\title{
Maslinic Acid Enhances Signals for the Recruitment of Macrophages and Their Differentiation to M1 State
}

\author{
Cristina Sánchez-Quesada, ${ }^{1,2,3}$ Alicia López-Biedma, ${ }^{1,2,3}$ and José J. Gaforio ${ }^{1,2,3}$ \\ ${ }^{1}$ Immunology Division, Department of Health Sciences, Faculty of Experimental Sciences, University of Jaen, \\ Campus las Lagunillas, s/n, 23071 Jaén, Spain \\ ${ }^{2}$ Centro de Estudios Avanzados en Olivar y Aceites de Oliva, Parque Científico-Tecnológico Geolit, c/ Sierra Morena, \\ Edificio CTSA Módulo 1, Mengíbar, 23620 Jaén, Spain \\ ${ }^{3}$ Agrifood Campus of International Excellence (ceiA3), University of Jaén, Campus Las Lagunillas, s/n, 23071 Jaén, Spain \\ Correspondence should be addressed to José J. Gaforio; jgaforio@ujaen.es
}

Received 6 November 2014; Revised 22 December 2014; Accepted 14 February 2015

Academic Editor: Shylaja M. Dharmesh

Copyright (C) 2015 Cristina Sánchez-Quesada et al. This is an open access article distributed under the Creative Commons Attribution License, which permits unrestricted use, distribution, and reproduction in any medium, provided the original work is properly cited.

\begin{abstract}
The inflammatory process is involved in the genesis and evolution of different diseases like obesity, cardiovascular disease, and cancer. Macrophages play a central role in inflammation. In addition, they can regulate some stages of cancer development. Macrophages can polarize into M1 or M2 functional phenotype depending on the cytokines present in the tissue microenvironment. On the other hand, triterpenes found in virgin olive oil are described to present different properties, such as antitumoral and anti-inflammatory activity. The present study was designed to elucidate if the four major triterpenes found in virgin olive oil (oleanolic acid, maslinic acid, uvaol, and erythrodiol) are able to enhance M1 macrophage response which represents an important defense mechanism against cancer. Our results indicated that maslinic acid modulated the inflammatory response by enhancing the production of IL- 8 , IL- $1 \alpha$, and IL- $1 \beta$; it promoted M1 response through the synthesis of IFN- $\gamma$; and finally it did not modify significantly the levels of $\mathrm{NF} \kappa \beta$ or NO. Overall, our results showed that maslinic acid could prevent chronic inflammation, which represents a crucial step in the development of some cancers.
\end{abstract}

\section{Introduction}

It is well known that patients with chronic inflammation are at a much higher risk of developing cancer. In 1863, Virchow hypothesized a link between inflammation and cancer based on the presence of leukocytes in neoplastic tissue [1]. In fact, the innate immune system, as a first line of defense, mediates the process of inflammation. In vitro and in vivo studies showed signals of inflammation in multiple pathways related to cancer development [2]. Since the evidences showed that immune cells are able to regulate almost every stage of cancer development, it would be interesting to explore biological mechanisms that could have the potential to modulate the immune response in order to reduce risks.

Macrophages play a central role in the development and maintenance of the inflammatory response. Furthermore, macrophages represent the predominant cellular type of the innate immune response found within tumors and are known as tumor-associated macrophages (TAMs). For example, breast cancer is characterized by having a large population of TAMs. Additionally, TAMs release factors to decrease the local proinflammatory antitumor response, suppressing it and providing a means of escape of the tumor cells [3].

These cells are able to synthesize a wide variety of molecules such as proinflammatory cytokines, antiinflammatory cytokines, or proteins related with the whole inflammation process such as nuclear factor kappa beta $(\mathrm{NF}-\kappa \beta)$, which in turn can trigger the synthesis of the proinflammatory cytokine IL- $1 \beta$. The activation of NF- $\kappa \beta$ into inflammatory response could be induced by other proinflammatory molecules like nitric oxide or by feedback of proinflammatory cytokines (IL-1, TNF- $\alpha$ ) [4]. TAMs come from monocytic precursors and undergo specific differentiation depending on local cues in the tissue. 


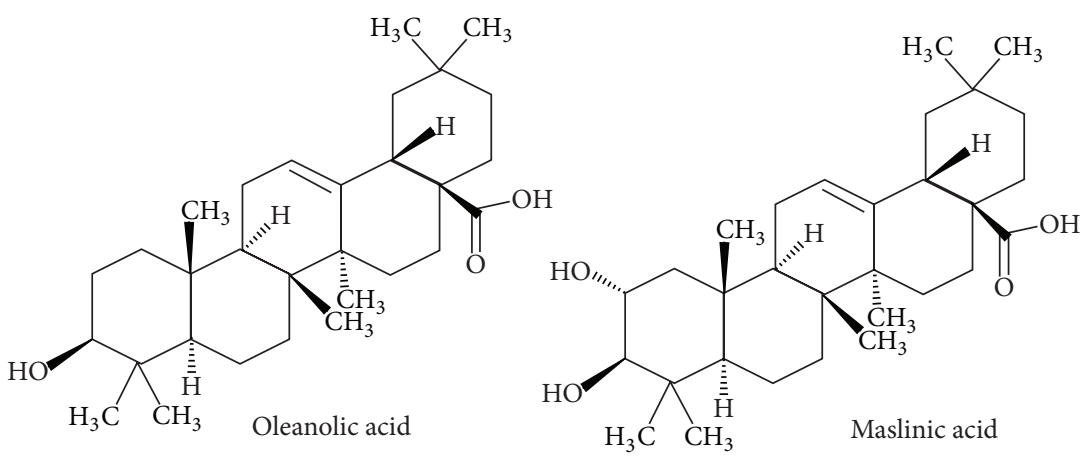

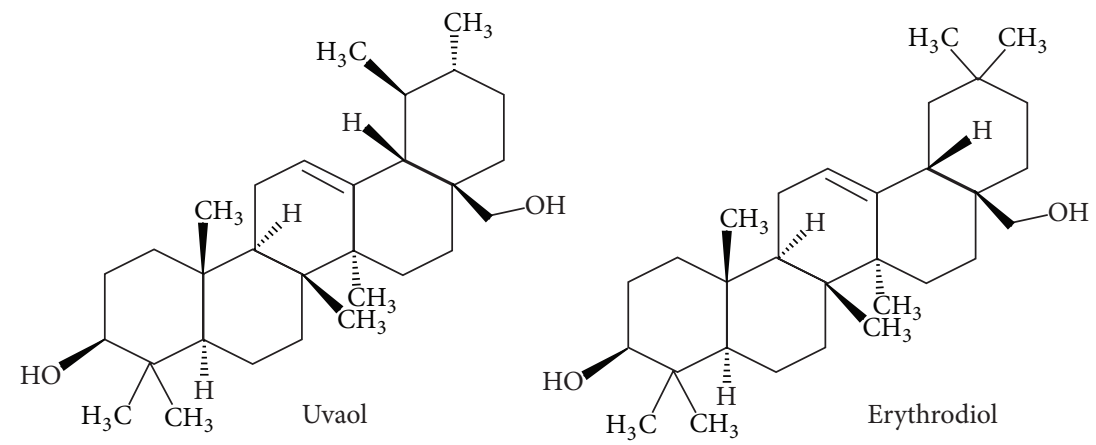

FIGURE 1: Chemical structure of oleanolic acid, maslinic acid, uvaol, and erythrodiol triterpenes.

Macrophages can be differentiated to M1 macrophages or M2 macrophages. M1 macrophages activate type 1 helper $\mathrm{T}$ cells (Th1), they are able to kill pathogens, and they are tumoricidal. On the other hand, M2 macrophages activate type 2 helper $\mathrm{T}$ cells (Th2), they are involved in wound healing where they downregulate the inflammatory reactions and promote angiogenesis, and they have a weak tumoricidal capability [5]. TAMs are often abundantly present in malignant tumors and share many common features with the alternative activated anti-inflammatory macrophages (M2). Furthermore, these cells have been shown to enhance tumor progression by promoting tumor invasion, migration, and angiogenesis. It is well established that, depending on the stage of tumor and the kind of macrophage population present, the tumor growth could be affected. As a matter of fact, in solid tumor a high M1/M2 ratio is associated with an improved survival [6]. Thus, it seems clear that a predominance of $\mathrm{M} 1$ macrophage response is beneficial to modulate the inflammatory response in carcinogenesis; it could act against cancer by promoting a Thl cytotoxic response.

On the other hand, oleanolic acid (OA), maslinic acid (MAS), uvaol (UV), and erythrodiol (ER) are the main pentacyclic triterpenes (Figure 1) found in both olive fruit and virgin olive oil [7], the principal source of fat on Mediterranean diet [8]. The benefits of the Mediterranean diet are well known $[9,10]$. It is believed that virgin olive oil is responsible for these beneficial effects, among other things, because of its anticarcinogenic properties and protection against DNA damages [11-15]. The main triterpenes of virgin olive oil have been described to possess cardioprotective activities
$[16,17]$, antitumor properties $[7,15]$, and anti-inflammatory activity [18-22]. These triterpenes are synthesized in the leaves and drupe of olive tree, and they are formed from the 2,3-oxidosqualene skeleton. The oleanolic and maslinic acids derive from the oleanane structure, while uvaol derives from ursane structure [23]. The difference between maslinic acid and oleanolic acid is an additional $\mathrm{OH}$ group in maslinic acid structure (carbon 2) (Figure 1).

There are no reports on the effect of triterpenes on M1 macrophage response. The present study was designed to investigate the effect of triterpenes found in virgin olive oil on $\mathrm{M} 1$ macrophage response.

\section{Materials and Methods}

2.1. Chemicals. Erythrodiol (ER) CAS [545-48-2], uvaol (UV) CAS [545-46-0], and oleanolic acid (OA) CAS [50802-1] (purity $\geq 97,98,5$, and 99\%, resp.) were purchased from Extrasynthese (Genay, France). Maslinic acid (MAS) CAS [4373-41-5] (purity >98\%) was obtained from Cayman Chemical (Ann Arbor, MI, USA). The following were purchased from Sigma-Aldrich Co. (St Louis, MO, USA): HEPES solution; sodium pyruvate solution; nonessential amino acids mixture 100× (NEAA); lipopolysaccharides from $E$. coli 055:B5 (LPS); 2,3-Bis(2-methoxy-4-nitro-5-sulfophenyl)2H-tetrazolium-5-carboxanilide inner salt (XTT sodium salt) (purity $\geq 90 \%$ ); N-methylphenazinium methyl sulfate (PMS) (purity $\geq 98 \%$ ); phorbol 12-myristate 13-acetate (PMA) 
(purity $\geq 99 \%$ ); phosphate buffer saline (PBS); sodium chloride $(\mathrm{NaCl})$ (purity $\geq 99,5 \%$ ); L-arginine (L-Arg) (purity 98.5$101.0 \%$ ) suitable for cell culture and Triton X-100. Fetal bovine serum (FBS) was obtained from PAA Laboratories $\mathrm{GmbH}$ (Pasching, Austria). Minimum essential medium with Eagle's salts (MEM) and phenol-red-free Roswell Park Memorial Institute 1640 medium (RPMI) were obtained from Gibco Life Technologies Ltd. (Paisley, UK). Methanol dry (maximum 0,005\%), magnesium chloride (50\% $\mathrm{MgCl}_{2}$ powder QP) $\left(\mathrm{MgCl}_{2}\right)$, and ethanol absolute were purchased from Panreac Quimica S.L.U. (Barcelona, SPAIN). TrypLE Express was obtained from Invitrogen (Eugene, OR, USA). $\beta$-Mercaptoethanol was purchased from Applichem $\mathrm{GmbH}$ (Darmstadt, GERMANY). PIPES (98,5+\%) was obtained from Acrōs Organics (Geel, Belgium). Culture plates were obtained from Starlab (Hamburg, Germany). NF $\kappa \beta$ p65 (F6) antibody was purchased from Santa Cruz Biotechnology (Santa Cruz, CA, USA). RayBio Human Cytokine Antibody Array (Human Inflammation Array I) was purchased from RayBiotech Inc. (Norcross, GA, USA).

2.2. Cell Line and Culture Conditions. The THP-1 (human acute monocytic leukemia) cell line was obtained from American Type Culture Collection (ATCC, Rockville, MD, USA). Cells were maintained at $37^{\circ} \mathrm{C}$ in a humidified atmosphere with $5 \% \mathrm{CO}_{2}$ in MEM supplemented with $10 \% \mathrm{FBS}, 1 \%$ HEPES buffer, $1 \%$ sodium pyruvate, $1 \%$ NEAA, and $0,05 \mathrm{mM}$ 2-mercaptoethanol. THP-1 cells were subcultured at least twice per week, discarded, and replaced by frozen stocks after 25 passages for achieving an optimal condition of growth.

Macrophages differentiation was induced by treating THP-1 cells $\left(1 \times 10^{6}\right.$ cells $\left./ \mathrm{mL}\right)$ for $24 \mathrm{~h}$ with $50 \mathrm{nM}$ of PMA followed by a period of further culture without PMA. PMA-differentiated THP- 1 cells $\left(1,5 \times 10^{5}\right.$ cells $\left./ \mathrm{mL}\right)$ were stimulated for $24 \mathrm{~h}$ with LPS $(1 \mu \mathrm{g} / \mathrm{mL})$ to acquire the M1 phenotype macrophage, and it was followed by oleanolic acid (OA), maslinic acid (MAS), uvaol (UV), or erythrodiol (ER) treatment at 1,10 , and $100 \mu \mathrm{M}$ for $4 \mathrm{~h}$. All the assays were conducted under these conditions except for those specified below.

2.3. Cytotoxicity Assay. THP-1 cells survival, measured as the cellular growth of treated cells versus untreated cells, was carried out using an XTT-based assay according to Warleta et al. [13]. Briefly, cells were seeded into 96-well culture plates in a total volume of $100 \mu \mathrm{L}$ per well. After overnight incubation to allow cell attachment, fresh medium was added with triterpenes in a range of concentrations from $0,001 \mu \mathrm{M}$ to $100 \mu \mathrm{M}$ of OA, MAS, UV, or ER for $24 \mathrm{~h}$. Thereafter, cells were incubated with XTT in phenol-red-free RPMI medium for $3 \mathrm{~h}$, and absorbance was measured at $450 \mathrm{~nm}$ wavelength (620 $\mathrm{nm}$ as reference) in a plate reader (TECAN GENios Plus). Viability was calculated using the following formula:

$$
\% \text { viable cells }=\left[\frac{(A \text { treated cells })}{(A \text { control })}\right] \times 100,
$$

where $A$ is the difference in absorbance between optical density units $\left(A=\mathrm{OD}_{450}-\mathrm{OD}_{620}\right)$. All measurements were performed in quadruplicate and each experiment was repeated at least three times.

2.4. RayBio Human Cytokine Antibody Array in M1 State THP1 Macrophages. Differentiated THP-1 cells were stimulated with LPS $(1 \mu \mathrm{g} / \mathrm{mL})$ for $24 \mathrm{~h}$. After that, cells were treated with triterpenes. A negative control (cells undifferentiated and untreated) was also tested. Then, supernatants were isolated and processed according to manufacturer instructions. Arrays membranes were directly detected using a chemiluminescence imaging system (FluorChem E System, ProteinSimple) to achieve production levels of the following cytokines/proteins: eotaxins, eotaxin-2, interleukin-1 alfa (IL-1 $\alpha$ ), interleukin-1 beta (IL-1 $\beta$ ), interleukin-2 (IL-2), interleukin-3 (IL-3), interleukin-4 (IL-4), interleukin-6 (IL6), interleukin-7 (IL-7), interleukin-8 (IL-8), interleukin10 (IL-10), interleukin-11 (IL-11), interleukin-12 p40 (IL12 p40), interleukin-12 p70 (IL-12p70), interleukin-13 (IL13), interferon-gamma (IFN-gamma), granulocyte colonystimulating factor (GCSF), granulocyte macrophage colonystimulating factor (GMCSF), chemokine CCL-1 (I-309), and metallopeptidase inhibitor 2 (TIMP-2).

Data were analyzed with the RayBio Human Inflammation Antibody Array 1 Analysis Tool (Catalogue number SO2AAH-INF-1). Data are expressed as the relative intensity (RI) between the sample and the LPS stimulated control [RI = $\left.\left(\mathrm{AU}_{\text {sample }} / \mathrm{AU}_{\text {control }}\right)\right]$, where $\mathrm{AU}$ is the chemiluminescence arbitrary units acquired by the chemiluminescence imaging system.

The results are showed like the fold change (ratio of the sample value respect to the control, which was set as 1).

\subsection{Flow Cytometry for NFא $\beta$ Detection in M1 State THP-1} Macrophages. After stimulation of differentiated THP-1 cells with LPS $(1 \mu \mathrm{g} / \mathrm{mL})$, cells were treated with OA, MAS, UV, and ER at 1, 10, and $100 \mu \mathrm{M}$. Cells were harvested with TrypLE Express and centrifugated at $300 \times \mathrm{g}$ at $4^{\circ} \mathrm{C}$ for $10 \mathrm{~min}$. The supernatant was discarded and $150 \mu \mathrm{L}$ of methanol was added. Cells were incubated $10 \mathrm{~min}$ at $-20^{\circ} \mathrm{C}$ and washed with PBS. Then, $1 \mathrm{~mL}$ of PIPES buffer (PIPES $10 \mathrm{mM}, \mathrm{NaCl} 0,1 \mathrm{M}$, $\mathrm{MgCl}_{2} 2 \mathrm{mM}$, and $0,1 \%$ Triton X100 on PBS) was added to each tube. Cells were incubated at room temperature (RT) for $10 \mathrm{~min}$. After that, cells were washed and suspended in anti-NF $\kappa \beta$ antibody buffer $(1 \mu \mathrm{g} / 100 \mu \mathrm{L})$ on darkness at RT for $30 \mathrm{~min}$. Later, cells were washed and analyzed by flow cytometry (EPICS XL-MCL, Beckman Coulter, Spain). NF$\kappa \beta$ production was calculated using the FlowJo program (v5.7.2). Each experiment was repeated at least three independent times. Data are represented as percentage of production of $\mathrm{NF} \kappa \beta$ with respect to control, which was set as $100 \%$.

2.6. NO Production in M1 Type THP-1 Macrophages. Nitric oxide (NO) production was measured according to F. Amano with some modifications [24]. Differentiated THP-1 cells $(5 \times$ $10^{5}$ cells $/ \mathrm{mL}$ ) were seeded on a 12 -well plate and treated with OA, MAS, UV, or ER at $0.1,1$, and $10 \mu \mathrm{M}$ for $3 \mathrm{~h}$. Then, LPS $(1 \mu \mathrm{g} / \mathrm{mL})$ and L-arginine (L-Arg) at $10 \mathrm{mM}$ were added to cells and incubated for $24 \mathrm{~h}$. Supernatants were 


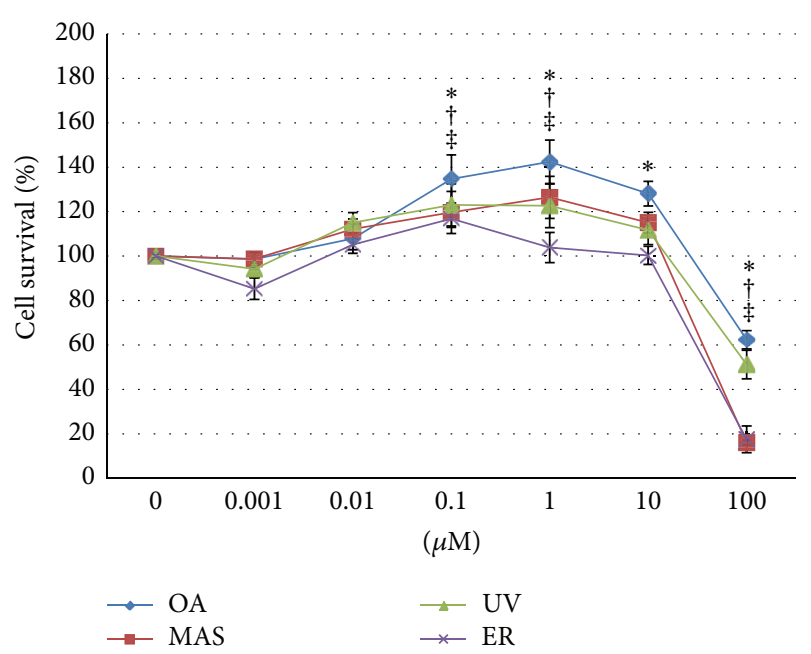

FIGURE 2: Effects of triterpenes on THP-1 macrophages survival. Cells were differentiated and treated with 0.001-0.01-0.1-1-10-100 $\mu \mathrm{M}$ of oleanolic acid $\left(\mathrm{OA}^{*}\right)$, maslinic acid $\left(\mathrm{MAS}^{\dagger}\right)$, uvaol $\left(\mathrm{UV}^{\ddagger}\right)$, and erythrodiol $\left(\mathrm{ER}^{\Delta}\right)$ at $24 \mathrm{~h} .{ }^{(*)(\dagger)(\ddagger)(\Delta)}$ Statistically significant differences compared with cells untreated $(P<0.05)$.

collected and incubated with ethanol absolute $30 \mathrm{~min}$ at $-20^{\circ} \mathrm{C}$. Supernatants were centrifuged at $10.000 \times \mathrm{g}$ at $4^{\circ} \mathrm{C}$ for $10 \mathrm{~min}$ and they were aliquoted. Production of $\mathrm{NO}$ was measured by a NO analyzer (NOA 280i de SIEVERS, GE Water and Process Technologies, Pennsylvania, USA). Data are expressed as the percentage of $\mathrm{NO}$ detection relative to untreated control, which was set as $100 \%$.

2.7. Statistical Analysis. For all the assays except for cytokine antibody array, data are displayed as the mean of at least three independent experiments $( \pm S E M)$; for cytotoxicity assay, results are expressed as a percentage relative to the untreated control cells (which was defined as 100\%). A general variance analysis (ANOVA) was carried out on data followed by Fisher's LSD test. A $P$ value $<0.05$ was considered to be statistically significant. These statistical analyses were performed using Statgraphics Centurion XVI statistical software (Statpoint Technologies Inc., Warrenton, VA).

\section{Results}

3.1. Cytotoxicity Effects. Cell survival was determined by the XTT assay. THP-1 cells were differentiated and exposed to increasing concentrations (from $0,001 \mu \mathrm{M}$ to $100 \mu \mathrm{M}$ ) of OA, MAS, UV, and ER for $24 \mathrm{~h}$. Our results showed that the four triterpenes assayed decreased significantly cell viability at $100 \mu \mathrm{M}$, whereas, at low concentrations, they did not show cytotoxic effects (Figure 2).

3.2. RayBio Human Cytokine Antibody Array. Production of inflammation-related proteins was measured on THP-1 macrophages cells stimulated with LPS $(1 \mu \mathrm{g} / \mathrm{mL})$ for differentiation of M1 phenotype. All the inflammation-related proteins showed significant differences in LPS stimulated cells with respect to untreated cells (Figures 3(a) and 4(a)).
3.2.1. M1/M2 Polarization Related Cytokines. After triterpenic treatments we observed that IFN- $\gamma$ level, which leads to M1 polarization, was increased with respect to control at MAS $1 \mu \mathrm{M}, 10 \mu \mathrm{M}$ and ER $1 \mu \mathrm{M}$ (Figures 3(b) and 3(c)). For the rest of compounds, IFN- $\gamma$ production levels were similar to control (Figures 3(d) and 3(e)). However, IL-4, which leads to M2 polarization, decreased levels after MAS $1 \mu \mathrm{M}$ treatment and is absent after MAS $10 \mu \mathrm{M}$, ER $1 \mu \mathrm{M}$, and $10 \mu \mathrm{M}$ treatment (Figures 3(b) and 3(c)). IL-10 did not show any significant differences with respect to control. In the other triterpenes tested there were not differences with respect to control (Figures 3(d) and 3(e)). At the concentration of $100 \mu \mathrm{M}$, most of the compounds have strong differences with respect to the control, but it might be due to the cytotoxic effects that they exerted at elevated concentrations.

3.2.2. Macrophages Recruitment-Related Cytokines and Proinflammatory Cytokines. Cytokines related with macrophages recruitment such as IL-8, IL-1 alpha, and IL-1 beta appeared increased in macrophages after treatment of MAS at $10 \mu \mathrm{M}$. The production of IL- 6 cytokine increased at the same concentration (Figure 4(b)). For the rest of compounds only the IL-8 cytokine production was increased at UV $10 \mu \mathrm{M}$ and IL- 1 alpha at ER $10 \mu \mathrm{M}$. The IL- 6 cytokine levels were increased in all the treatments at $10 \mu \mathrm{M}$ and at ER $1 \mu \mathrm{M}$ (Figures 4(c), 4(d), and 4(e)).

For the rest of cytokines and proteins related with inflammation, the signals were closed to background (data not shown).

3.3. Effects on NF- $\kappa \beta$ Production. Detection of NF- $\kappa \beta$ (p65) was performed by flow cytometry in differentiated THP-1 cells stimulated with LPS $24 \mathrm{~h}$ and treated with 1,10 , and $100 \mu \mathrm{M}$ of OA, MAS, UV, or ER triterpenes. There were not statistically significant differences between control and samples (Table 1).

3.4. NO Production. NO production was measured on M1 phenotype THP-1 macrophages at $0.1,1$, and $10 \mu \mathrm{M}$ of OA, MAS, UV, or ER. Although any treatment exhibited a statistically significant variation compared with the LPS stimulated control, a slight increase of NO production was observed at MAS $1 \mu \mathrm{M}, 10 \mu \mathrm{M}$, and ER $10 \mu \mathrm{M}$ and a decrease at OA $10 \mu \mathrm{M}$ and ER $1 \mu \mathrm{M}$ (Figure 5). LPS stimulated control showed statistical differences with respect to unstimulated control (data not shown).

\section{Discussion}

The THP-1 cell line has a closed gene expression to primary macrophages, derived from peripheral blood mononuclear cells, in contrast to other monocytes cell lines like U937 [25]. Furthermore, a PMA differentiation of THP-1 cells drives cells to a differentiated macrophage phenotype that seems very nearby to monocyte-derived human macrophages [26]. Analysis of primary macrophages in culture will always provide more truthful information about inflammation response 


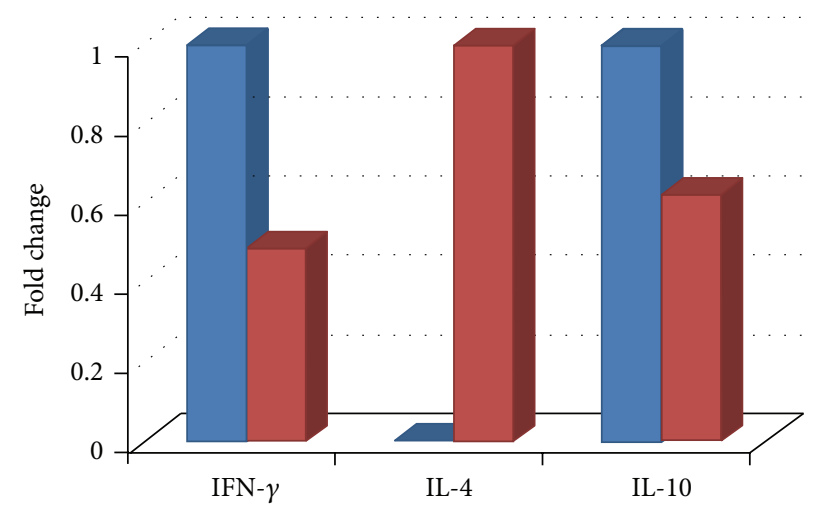

$\square$ Cells untreated

- LPS treated

(a)

MAS

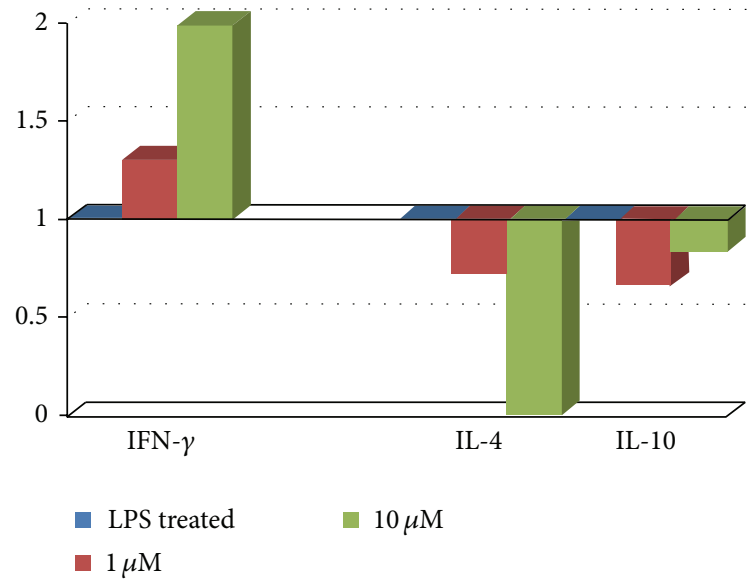

(b)

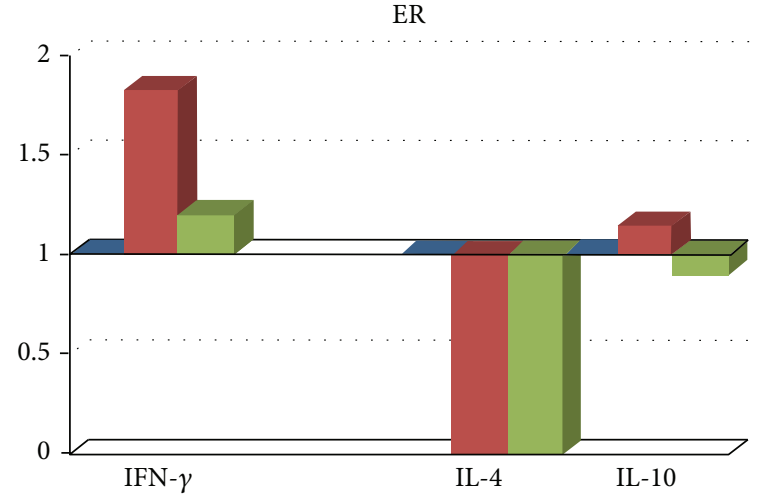

- LPS treated $\quad 10 \mu \mathrm{M}$

$\square \mathrm{M}$

(c)

$\mathrm{OA}$

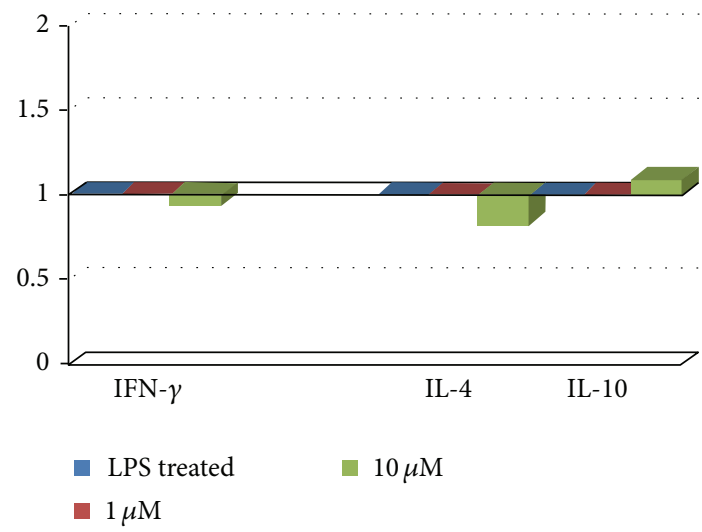

(d)

UV

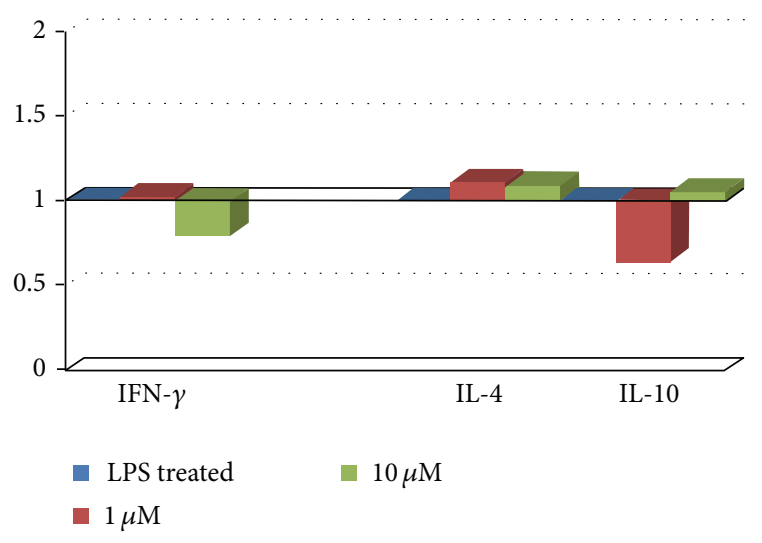

(e)

FIGURE 3: M1 polarization related cytokines production versus M2 polarization related cytokines production in cell unstimulated and stimulated with LPS (a) and in M1 polarized THP-1 macrophages after treatment with MAS (b), ER (c), OA (d), and UV (e) at 1, 10, and $100 \mu \mathrm{M}$. Results are expressed as the fold change in RI (relative intensity) related to stimulated control which was set as 1. 


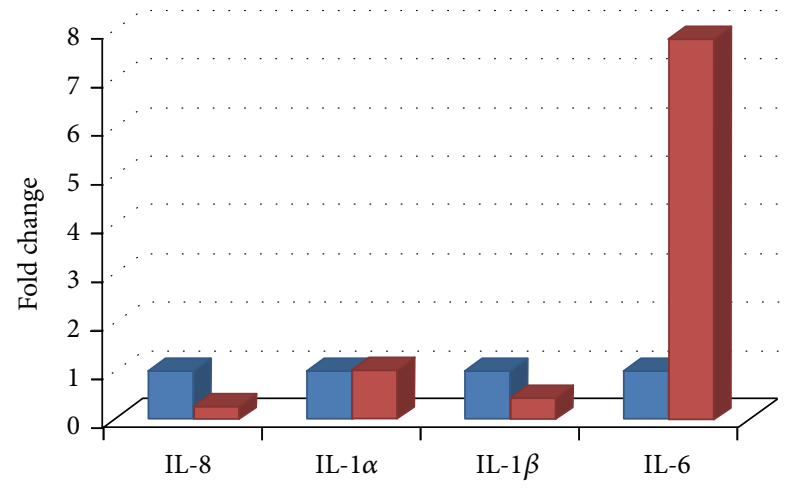

Cells untreated

- LPS treated

(a)

MAS

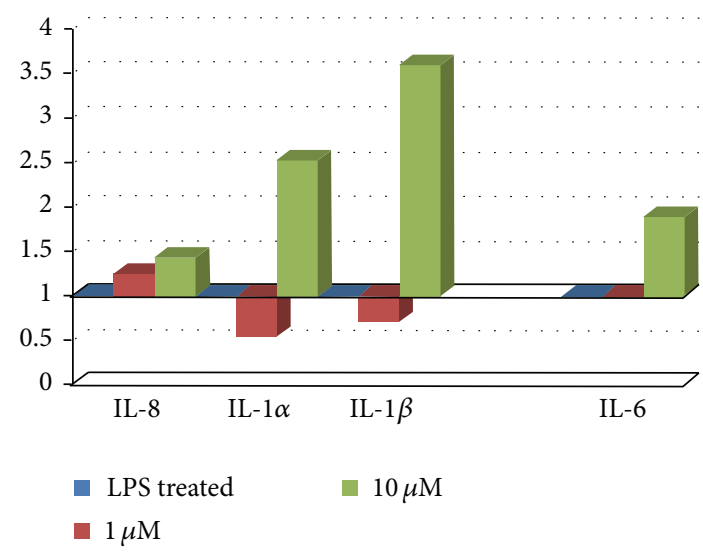

(b)

UV

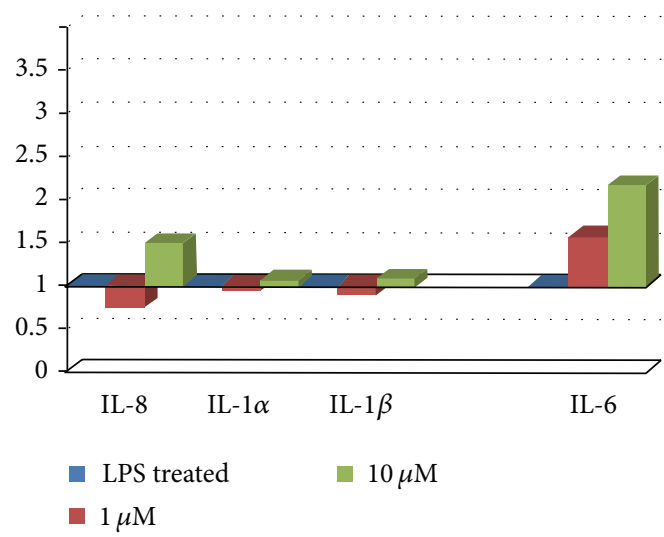

(d)

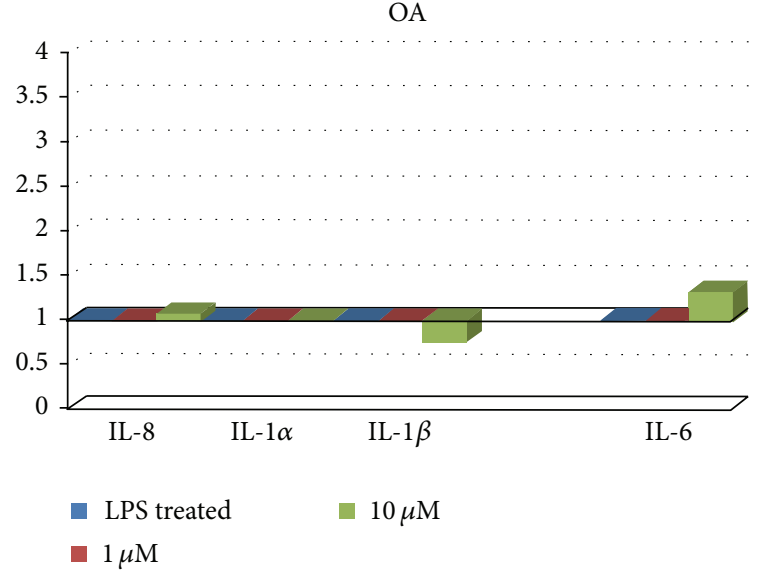

(c)

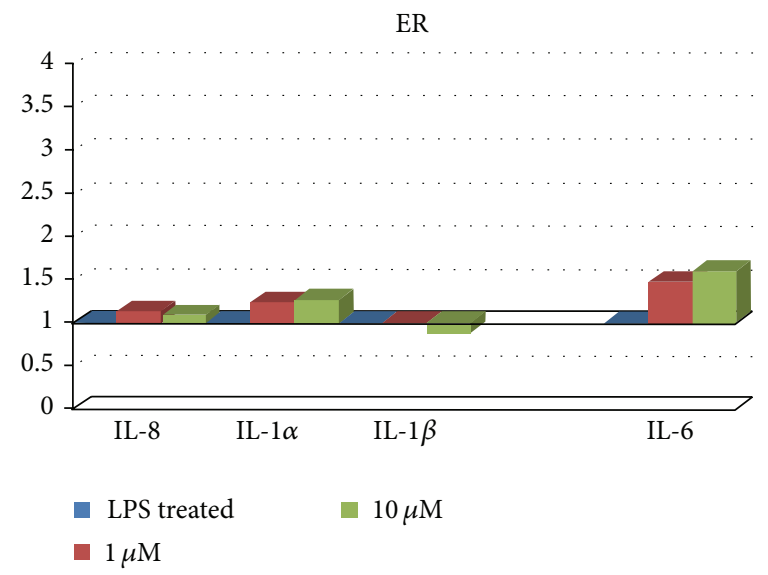

(e)

FIGURE 4: Production of cytokines responsible of macrophage recruitment produced in cell unstimulated and stimulated with LPS (a) and in M1 polarized THP-1 macrophages treated with MAS (b), OA (c), UV (d), and ER (e) at 1, 10, and $100 \mu \mathrm{M}$.

than cellular models, but these primary cultures are also difficult to culture in the quantities required to allow biochemical analysis. Thus, PMA differentiated and LPS stimulated THP1 cells represent a useful experimental model to study the inflammatory response and their modulation after treatment with food compounds [27]. Moreover, recently the consequent polarization to M1 phenotype that LPS promotes in THP-1 macrophages has been described [28]. Thus, THP-1 
TABLE 1: NF $\kappa \beta$ production by M1 polarized THP-1 macrophage cells treated with OA, MAS, UV, and ER at 1,10 , and $100 \mu \mathrm{M}$ along $4 \mathrm{~h}$, measured by flow cytometry. Data are expressed like the percentage of $N F \kappa \beta$ production with respect to cells LPS treated, which was set as $100 \%$. Standard error means (SEM) represented as percentage. Not statistical differences found at $P<0.05$.

\begin{tabular}{lccc}
\hline Treatment & Concentration & Mean & SEM \\
\hline LPS treated & & 100 & $\pm 66,05$ \\
\hline \multirow{3}{*}{ OA } & $1 \mu \mathrm{M}$ & 132 & $\pm 12,06$ \\
& $10 \mu \mathrm{M}$ & 74 & $\pm 17,15$ \\
& $100 \mu \mathrm{M}$ & 84 & $\pm 15,74$ \\
\multirow{2}{*}{ MAS } & $1 \mu \mathrm{M}$ & 96 & $\pm 13,26$ \\
& $10 \mu \mathrm{M}$ & 101 & $\pm 17,16$ \\
& $100 \mu \mathrm{M}$ & 116 & $\pm 30,50$ \\
\multirow{2}{*}{ UV } & $1 \mu \mathrm{M}$ & 112 & $\pm 19,54$ \\
& $10 \mu \mathrm{M}$ & 121 & $\pm 29,72$ \\
& $100 \mu \mathrm{M}$ & 102 & $\pm 23,86$ \\
\multirow{2}{*}{ ER } & $1 \mu \mathrm{M}$ & 110 & $\pm 38,91$ \\
& $10 \mu \mathrm{M}$ & 101 & $\pm 28,62$ \\
& $100 \mu \mathrm{M}$ & 99 & $\pm 23,88$ \\
\hline
\end{tabular}

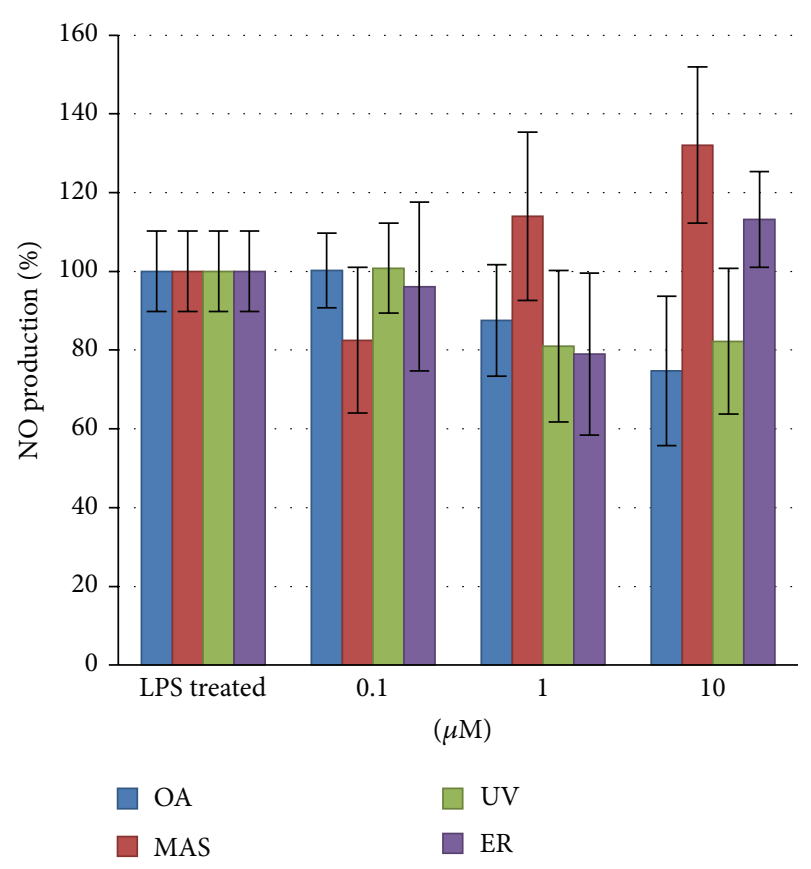

FIGURE 5: NO production of M1 polarized THP-1 macrophages treated with $0.1,1$, and $10 \mu \mathrm{M}$ of OA, MAS, UV, and ER. Data are expressed relative to LPS treated cells, which was established as $100 \%$. Not statistical differences were found.

macrophages are the best option for study in vitro effects of certain compounds in macrophages differentiated to M1 phenotype.

According to this, THP-1 cells were used to study in vitro effects of OA, MA, UV, and ER on M1 macrophages. THP1 macrophages were differentiated into the M1 stage by LPS treatment.
Macrophages constitute an extremely heterogeneous population, which polarize into distinct macrophages types, mainly identified as M1 (or classically activated) and M2 (or alternatively activated) [29]. Previously, our group described two phenotypically and functionally different populations among splenic macrophages in response to C. albicans infection. One of them (M2 phenotype) expressed high levels of major histocompatibility complex (MHC) class II surface expression and is poorly phagocytic. The other one (M1 phenotype) expressed low levels of MHC class II molecules and is highly phagocytic [30]. We suggested that NK cells prime splenic macrophages were phagocytic in naïve BALB/c mice, probably mediated by IFN $-\gamma$ production, the same signal that monocytes need in tumor microenvironment for polarized to M1 phenotype. Thus, infections as well as cancer could polarize macrophages to M1 or M2 phenotypes depending on the microenvironment signals [6].

In nonprogressing or regressing tumors, TAMs are related to a classic macrophage activation M1-like program, characterized by proinflammatory activity, antigen presentation, and tumor lysis. Even more, a high M1/M2 polarization ratio improved survival in lung carcinoma [6]. In malignant tumors, TAMs resemble M2 phenotype. These macrophages increase angiogenesis, tumor cell extravasation, and growth; they suppress activation of dendritic cells, cytotoxic T lymphocytes, and natural killers [31,32].

M1 macrophages appear to have a proangiogenic function early in tumorigenesis [33], when the tumor needs blood vessels formation to grow; this fact supports the idea of the role that M1 plays in the early stages of breast tumor formation and it seems to be one of the first immune cells present in the inflammatory process. But, in advanced breast cancers, macrophages resemble the M2 phenotype, while M1 phenotype has not been found; this is the reason why TAMs are generally related more to a M2 phenotype than M1. Further, M2 macrophages express changes in several metabolic pathways, controlling the inflammatory response by downregulating M1-mediated functions. It seems that tumor cells are able to produce several signals that polarize monocytes to M2. This preferential polarization is the result of absence of M1-orienting signals, such as INF- $\gamma$ or bacterial components in the tumor [29].

We hypothesized that, in established solid tumors, the activation of a M1 response could be a useful strategy in order to prevent tumor growth.

Our results show that MAS and ER at low concentration increased the production of INF- $\gamma$ in M1 polarized THP-1 macrophages. By this way, M1 macrophages could mediate and control their own response differentiating monocytes to M1 instead of M2 in carcinogenesis. This increase of INF- $\gamma$ production would be a proinflammatory signal for monocytes in inflammation locations, differentiating these monocytes to M1 phenotype (Figure 6), making more efficient the recognition of neoplastic cells, and mediating an effective Th1 cells response.

On the other hand, M2 macrophages can switch to M1 at the site of the tumor by INF- $\gamma$ induction and receptormediated activation signals to promote tumor regression [6]. With MAS and ER, it could be M1 polarized macrophages 


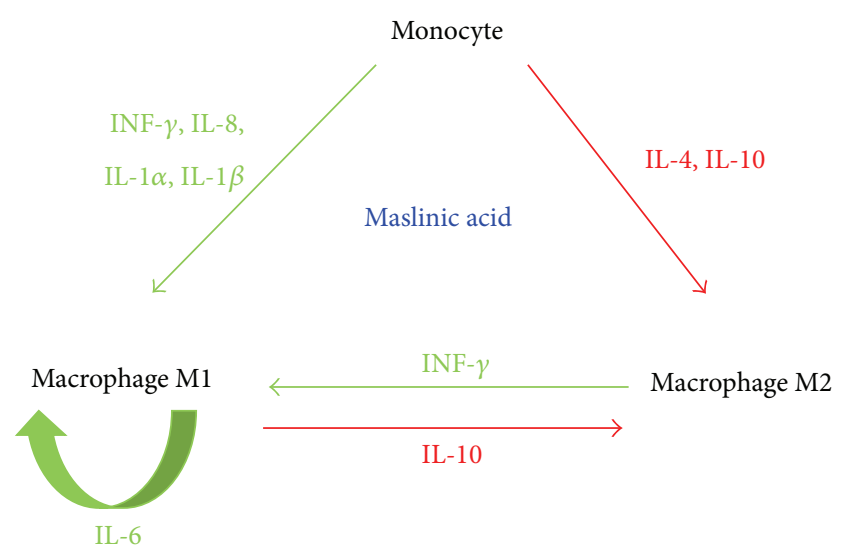

FIGURE 6: Role of maslinic acid in differentiation and recruitment of macrophages. Cytokine produced after MA treatment in M1 macrophages is represented in green and cytokine inhibited after MA treatment in M1 macrophages is represented in red.

theirselves, which could hypothetically reprogram the M2 macrophages that could show up at the sites of tumor formation, to M1 state.

Indeed, MAS at $10 \mu \mathrm{M}$ and $\mathrm{UV}$ at $10 \mu \mathrm{M}$ showed levels of IL-8 production slightly higher than the control (Figures 4 (b) and 4(d)). Although IL-8 production was not statistically significant, IL- $1 \alpha$ and IL- $1 \beta$ were dramatically increased. It is known that the role of IL- 8 cytokine on the monocyte recruitment as well as CXCL12 chemokine, whose precursors are IL-1 $\alpha$ and IL- $1 \beta$, interestingly increased in MAS at $10 \mu \mathrm{M}$ (Figure 4(b)).

Apart from fortifying the proinflammatory response by activating monocytes to $\mathrm{M} 1$ and preventing the M2 polarization of monocytes at sites of inflammation, MA appears to promote the recruitment of more cells that could support the immune response on the inflammation location.

Furthermore, some authors describe that NF- $\kappa \beta$ promotes the presence of immunosuppressive M2 phenotype [34]. In order to assess the possible role of triterpenes in promoting production of NF- $\kappa \beta$, we studied its production by the M1 phenotype THP-1 macrophages. It is important to note that a high increase of NF- $\kappa \beta$ expression may lead to an aggravated inflammatory response that could guide to a consequent chronic inflammation [34]. In our study, levels of NF- $\kappa \beta$ were not statically significant with respect to control untreated, so these M1 macrophages treated with triterpenes did not show to promote chronic inflammation.

Furthermore, nitric oxide (NO) production by NOS (nitric oxide synthase) supports this point. At normal levels, NOS acts like protector against injury, but at elevated levels in the tissue it has been described like an inflammatory enzyme that promotes carcinogenesis [35]. We studied the levels of NO after treatment with triterpenes and there was no statistically significant change in their production compared to the control. These results agree with those of MárquezMartín et al., who described an inhibition of NO production in peritoneal murine macrophages upon exposure to MA treatment [36]. At $0.1 \mu \mathrm{M}$ we notify the reduction of $\mathrm{NO}$ after MA treatment in M1 macrophages.

It is important to note that, at the highest concentration, these triterpenes are cytotoxic for THP-1 macrophages but the effects of these triterpenes in M1 macrophages focus at low concentrations.

\section{Conclusion}

Maslinic acid possesses two principal actions on M1 macrophages: first, it enhanced recruitment of macrophages by production of IL-8, IL-1 $\alpha$, and IL-1 $\beta$; and second, it promoted M1 response through the synthesis of IFN- $\gamma$.

Further studies are needed for assessing the action of these macrophages treated with triterpenes in carcinogenesis. However, maslinic acid could be a useful natural compound to modulate inflammation response.

\section{Conflict of Interests}

The authors declare that there is no conflict of interests regarding the publication of this paper.

\section{Acknowledgments}

This study was financially supported by the "Junta de Andalucía" (Proyecto de Excelencia PI10-AGR-6724) and supported by grants from University of Jaén (Plan de Apoyo a la Investigación-Acción 16).

\section{References}

[1] H. David, "Rudolf Virchow and modern aspects of tumor pathology," Pathology-Research and Practice, vol. 183, no. 3, pp. 356-364, 1988.

[2] S. Demaria, E. Pikarsky, M. Karin et al., "Cancer and inflammation: promise for biologic therapy," Journal of Immunotherapy, vol. 33, no. 4, pp. 335-351, 2010.

[3] D. Laoui, K. Movahedi, E. van Overmeire et al., "Tumorassociated macrophages in breast cancer: distinct subsets, distinct functions," International Journal of Developmental Biology, vol. 55, no. 7-9, pp. 861-867, 2011.

[4] B. B. Aggarwal, S. Shishodia, S. K. Sandur, M. K. Pandey, and G. Sethi, "Inflammation and cancer: how hot is the link?" Biochemical Pharmacology, vol. 72, no. 11, pp. 1605-1621, 2006.

[5] S. K. Biswas and A. Mantovani, "Macrophage plasticity and interaction with lymphocyte subsets: cancer as a paradigm," Nature Immunology, vol. 11, no. 10, pp. 889-896, 2010.

[6] M. Heusinkveld and S. H. van der Burg, "Identification and manipulation of tumor associated macrophages in human cancers," Journal of Translational Medicine, vol. 9, no. 1, article 216, 2011.

[7] Y. Allouche, F. Warleta, M. Campos et al., "Antioxidant, antiproliferative, and pro-apoptotic capacities of pentacyclic triterpenes found in the skin of olives on MCF-7 human breast cancer cells and their effects on DNA damage," Journal of Agricultural and Food Chemistry, vol. 59, no. 1, pp. 121-130, 2011.

[8] F. Perez-Jimenez, G. A. de Cienfuegos, L. Badimon et al., "International conference on the healthy effect of virgin olive 
oil," European Journal of Clinical Investigation, vol. 35, no. 7, pp. 421-424, 2005.

[9] A. Villarini, P. Pasanisi, A. Traina et al., "Lifestyle and breast cancer recurrences: the DIANA-5 trial," Tumori, vol. 98, no. 1, pp. 1-18, 2012.

[10] N. di Daniele, L. Petramala, L. di Renzo et al., "Body composition changes and cardiometabolic benefits of a balanced Italian Mediterranean diet in obese patients with metabolic syndrome," Acta Diabetologica, vol. 50, no. 3, pp. 409-416, 2013.

[11] R. Colomer and J. A. Menéndez, "Mediterranean diet, olive oil and cancer," Clinical and Translational Oncology, vol. 8, no. 1, pp. 15-21, 2006.

[12] R. W. Owen, R. Haubner, G. Würtele, W. E. Hull, B. Spiegelhalder, and H. Bartsch, "Olives and olive oil in cancer prevention," European Journal of Cancer Prevention, vol. 13, no. 4, pp. 319-326, 2004.

[13] F. Warleta, M. Campos, Y. Allouche et al., "Squalene protects against oxidative DNA damage in MCF10A human mammary epithelial cells but not in MCF7 and MDA-MB-231 human breast cancer cells," Food and Chemical Toxicology, vol. 48, no. 4, pp. 1092-1100, 2010.

[14] F. Warleta, C. S. Quesada, M. Campos, Y. Allouche, G. Beltrán, and J. J. Gaforio, "Hydroxytyrosol protects against oxidative DNA damage in human breast cells," Nutrients, vol. 3, no. 10, pp. 839-857, 2011.

[15] C. Sánchez-Quesada, A. López-Biedma, and J. J. Gaforio, “The differential localization of a methyl group confers a different anti-breast cancer activity to two triterpenes present in olives," Food \& Function, vol. 6, no. 1, pp. 248-255, 2015.

[16] S. Senthil, M. Sridevi, and K. V. Pugalendi, "Cardioprotective effect of oleanolic acid on isoproterenol-induced myocardial ischemia in rats," Toxicologic Pathology, vol. 35, no. 3, pp. 418423, 2007.

[17] Y. Allouche, G. Beltrán, J. J. Gaforio, M. Uceda, and M. D. Mesa, "Antioxidant and antiatherogenic activities of pentacyclic triterpenic diols and acids," Food and Chemical Toxicology, vol. 48, no. 10, pp. 2885-2890, 2010.

[18] R. Martín, M. Hernández, C. Córdova, and M. L. Nieto, "Natural triterpenes modulate immune-inflammatory markers of experimental autoimmune encephalomyelitis: therapeutic implications for multiple sclerosis," British Journal of Pharmacology, vol. 166, no. 5, pp. 1708-1723, 2012.

[19] V. S. Graham, C. Lawson, C. P. D. Wheeler-Jones, J. S. Perona, V. Ruiz-Gutierrez, and K. M. Botham, "Triacylglycerol-rich lipoproteins derived from healthy donors fed different olive oils modulate cytokine secretion and cyclooxygenase- 2 expression in macrophages: the potential role of oleanolic acid," European Journal of Nutrition, vol. 51, no. 3, pp. 301-309, 2012.

[20] C. Li, Z. Yang, C. Zhai et al., "Maslinic acid potentiates the antitumor activity of tumor necrosis factor alpha by inhibiting NFkappaB signaling pathway," Molecular Cancer, vol. 9, article 73, 2010.

[21] C. Sánchez-Quesada, A. López-Biedma, F. Warleta, M. Campos, G. Beltrán, and J. J. Gaforio, "Bioactive properties of the main triterpenes found in olives, virgin olive oil, and leaves of Olea europaea," Journal of Agricultural and Food Chemistry, vol. 61, no. 50, pp. 12173-12182, 2013.

[22] V. R. Yadav, S. Prasad, B. Sung, R. Kannappan, and B. B. Aggarwal, "Targeting inflammatory pathways by triterpenoids for prevention and treatment of cancer," Toxins, vol. 2, no. 10, pp. 2428-2466, 2010.
[23] N. Stiti, S. Triki, and M.-A. Hartmann, "Formation of triterpenoids throughout Olea europaea fruit ontogeny," Lipids, vol. 42, no. 1, pp. 55-67, 2007.

[24] F. Amano and T. Noda, "Improved detection of nitric oxide radical (NO) production in an activated macrophage culture with a radical scavenger, carboxy PTIO and griess reagent," FEBS Letters, vol. 368, no. 3, pp. 425-428, 1995.

[25] O. Sharif, V. N. Bolshakov, S. Raines, P. Newham, and N. D. Perkins, "Transcriptional profiling of the LPS induced NF- $\kappa \mathrm{B}$ response in macrophages," BMC Immunology, vol. 8, article 1, 2007.

[26] M. Daigneault, J. A. Preston, H. M. Marriott, M. K. B. Whyte, and D. H. Dockrell, "The identification of markers of macrophage differentiation in PMA-stimulated THP-1 cells and monocyte-derived macrophages," PLoS ONE, vol. 5, no. 1, Article ID e8668, 2010.

[27] W. Chanput, J. Mes, R. A. M. Vreeburg, H. F. J. Savelkoul, and H. J. Wichers, "Transcription profiles of LPS-stimulated THP1 monocytes and macrophages: a tool to study inflammation modulating effects of food-derived compounds," Food and Function, vol. 1, no. 3, pp. 254-261, 2010.

[28] W. Chanput, J. J. Mes, H. F. J. Savelkoul, and H. J. Wichers, "Characterization of polarized THP-1 macrophages and polarizing ability of LPS and food compounds," Food \& Function, vol. 4, no. 2, pp. 266-276, 2013.

[29] G. Solinas, G. Germano, A. Mantovani, and P. Allavena, "Tumor-associated macrophages (TAM) as major players of the cancer-related inflammation," Journal of Leukocyte Biology, vol. 86, no. 5, pp. 1065-1073, 2009.

[30] J. J. Gaforio, E. Ortega, I. Algarra, M. J. Serrano, and G. Alvarez de Cienfuegos, "Nk cells mediate increase of phagocytic activity but not of proinflammatory cytokine (interleukin-6 [IL6], tumor necrosis factor alpha, and IL-12) production elicited in splenic macrophages by tilorone treatment of mice during acute systemic candidiasis," Clinical and Diagnostic Laboratory Immunology, vol. 9, no. 6, pp. 1282-1294, 2002.

[31] A. Mantovani and A. Sica, "Macrophages, innate immunity and cancer: balance, tolerance, and diversity," Current Opinion in Immunology, vol. 22, no. 2, pp. 231-237, 2010.

[32] B.-Z. Qian and J. W. Pollard, "Macrophage diversity enhances tumor progression and metastasis," Cell, vol. 141, no. 1, pp. 3951, 2010.

[33] S. Samineni, Z. Zhang, and J. E. Shively, "Carcinoembryonic antigen-related cell adhesion molecule 1 negatively regulates granulocyte colony-stimulating factor production by breast tumor-associated macrophages that mediate tumor angiogenesis," International Journal of Cancer, vol. 133, no. 2, pp. 394-407, 2013.

[34] L. Jackson and B. M. Evers, "Chronic inflammation and pathogenesis of GI and pancreatic cancers," Cancer Treatment and Research, vol. 130, pp. 39-65, 2006.

[35] L. M. Coussens and Z. Werb, "Inflammation and cancer," Nature, vol. 420, no. 6917, pp. 860-867, 2002.

[36] A. Márquez Martín, R. de la Puerta Vázquez, A. FernándezArche, and V. Ruiz-Gutiérrez, "Supressive effect of maslinic acid from pomace olive oil on oxidative stress and cytokine production in stimulated murine macrophages," Free Radical Research, vol. 40, no. 3, pp. 295-302, 2006. 


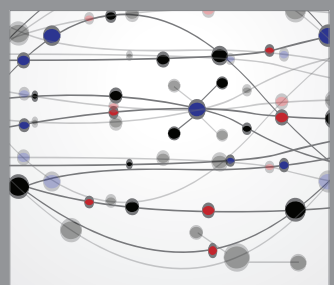

The Scientific World Journal
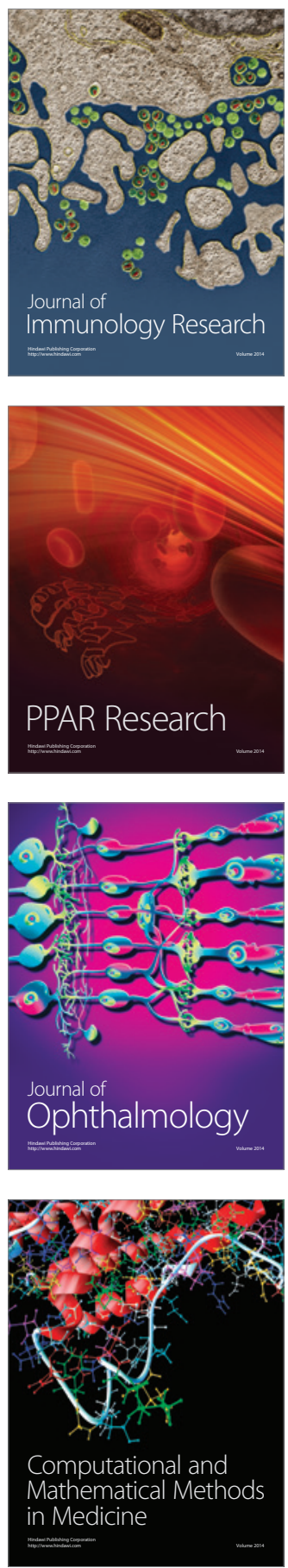

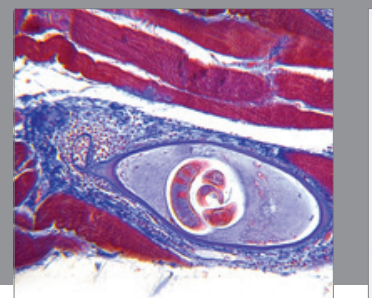

Gastroenterology

Research and Practice
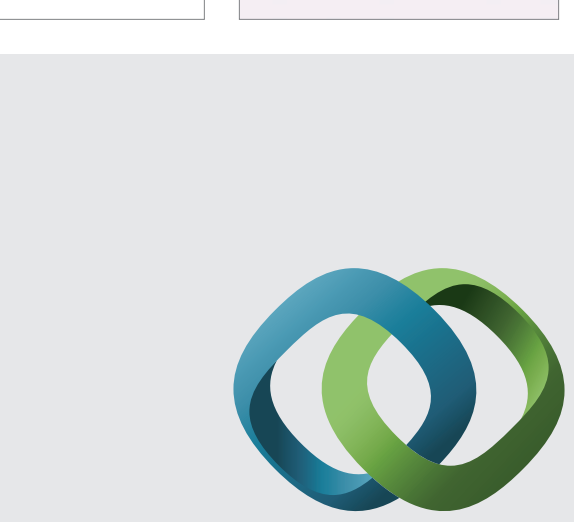

\section{Hindawi}

Submit your manuscripts at

http://www.hindawi.com
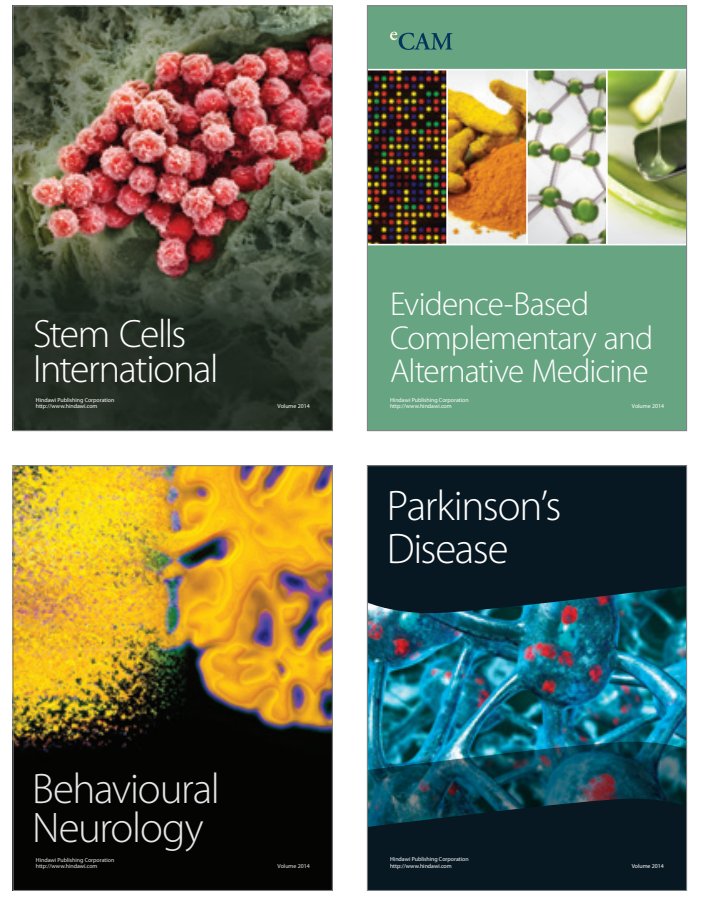
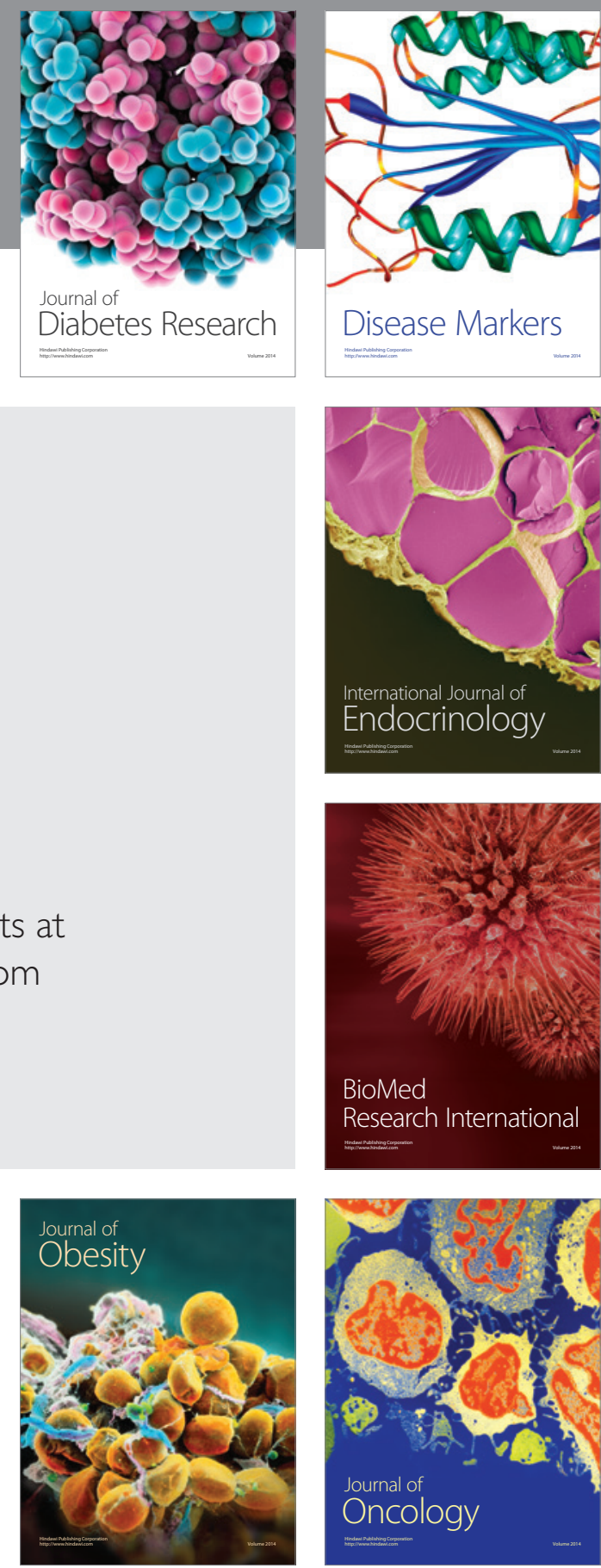

Disease Markers
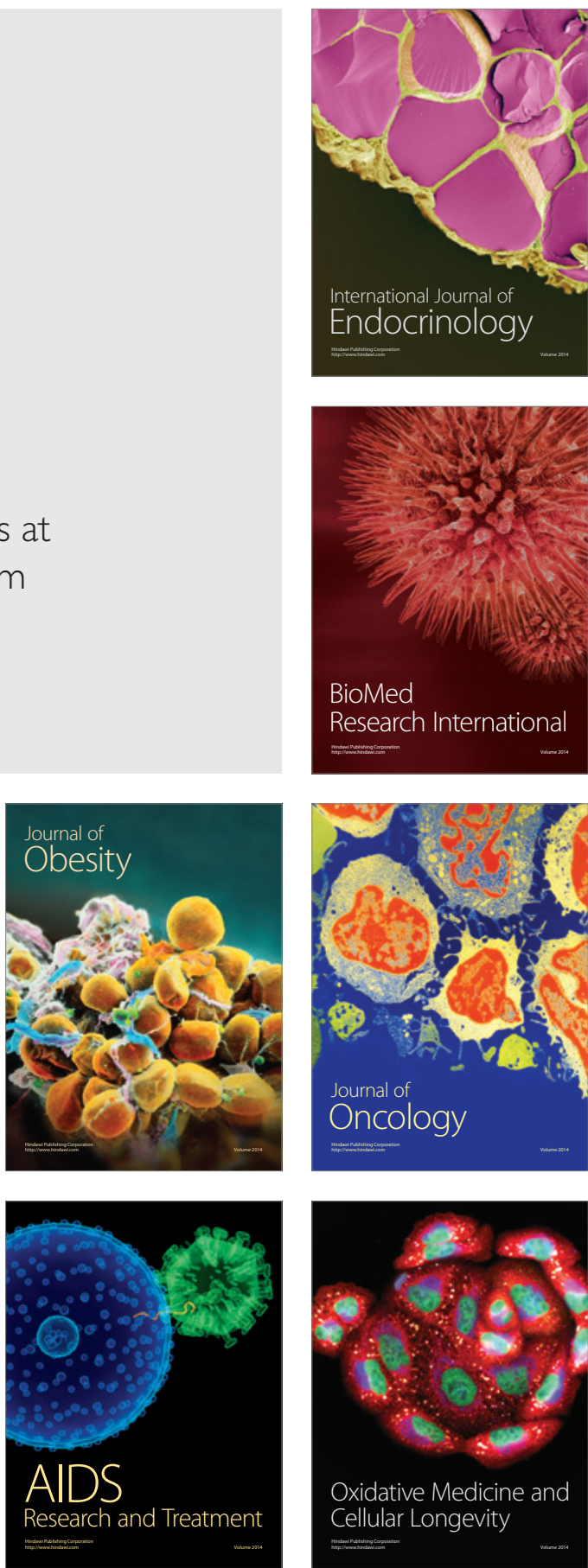